\title{
Detection of Combined Faults in Squirrel Cage Induction Motors
}

\author{
Kaikaa Mohamed Yazid ${ }^{1 *}$, Boudjelida Loubna² \\ 1'LGEC/University of Frere mentouri, Algeria; Email yazid.Kaikaa@gmail.com \\ ${ }^{2}$ LGEC/University of Frere mentouri, Algeria; Email Loubna_boudjelida@hotmail.fr
}

\begin{abstract}
The aim of this paper is to analyze theoretically and experimentally the stator current of an induction machine operating with mixed faults: broken rotor bars and dynamic eccentricity (DE). An analytical explanation of the generation mechanism of all frequencies related to the simultaneous presence of these two defects is given. It is demonstrated that DE diagnosis is very difficult if only stator current analysis is used. Experimental results have been presented to validate the analytical and simulation results.
\end{abstract}

Keywords: Broken rotor bars, fault diagnosis, induction machine, mixed faults, dynamic eccentricity.

\section{INTRODUCTION}

Broken rotor bars and dynamic eccentricity constitute a considerable portion of the faults related to squirrel cage induction motor. Rotor bar faults are usually associated with high temperatures or high mechanical loading. Dynamic eccentricity (DE) occurs when the center of the rotor is not at the centre of the rotation, and the position of minimum air-gap rotates with the rotor. Early detection of these faults can reduce the maintenance costs and prevent unscheduled downtime of the motor. This has spurred researchers, resulting in the development of many condition monitoring techniques. However, the most popular technique is based on the analysis of stator current (MCSA) [1].

The broken bar effects on the motor stator current were largely treated in the literature. It was shown that characteristics components at frequencies described in (1) appear in the stator current spectrum [2] [3], [4]:

$$
\begin{aligned}
& \quad f_{b b}(n)=(n(1-s) \pm s) f_{s} \\
& \text { Where, } n=1,5,7 \ldots \ldots 6 \eta \pm 1
\end{aligned}
$$

On the other hand, there have been also a number of papers dealing the effect of air gap eccentricity on the stator currents [5],[6],[7] ],[8].Using MCSA, the equation that describes the frequency components associated with a $(\mathrm{DE})$ is:

$$
f_{d e}=\left(\frac{\lambda n_{r} \pm n_{d}}{p}(1-s) \pm 1\right) f_{s}
$$

Where $f_{s}$ :voltage supply frequency, $p$ is the number of pole pairs, $n_{r}$ is the number of rotor bars, $s$ is the slip and $n_{d}=1,2$.

In practice, it is possible that broken rotor bars and DE occur simultaneously. To the others knowledge, this kind of mixed fault has not been analyzed in the literature yet. For that reason and since a reliable diagnosis requires a good understanding of fault signatures generation process, this paper proposes a new approach to investigate the generated harmonic components due to the coexistence of broken rotor bars and DE. Hence, this paper is organized as follows: in section 2, a theoretical approach is developed to calculate the harmonic components induced in the rotor cage currents due to the combined faults. Then, the study is extended to calculate the harmonic content of the stator current, in section 3. The verification of the theoretical analysis and computer simulations through experiments is presented in section 4 .

\section{GENERATION PROCESS OF HARMONICS IN ROTOR CAGE CURRENTS IN PRESENCE OF DE AND BROKEN ROTOR BARS}

The knowledge of a precise expression of the mutual inductance between stator windings and rotor loops is the key of this

*Corresponding authour

Email: yazid.Kaikaa@gmail.com (K.M. Yazid) 
research. It allows one to complete the analytical calculation of frequency components of both rotor and stator currents for machine with combined faults. According to [10] inductance between windings " $\mathrm{s}$ " and " $\mathrm{r}$ " in presence of DE can be computed by the following equation:

$$
\begin{aligned}
{\left[M_{s r}(\theta)\right] } & =M_{s r \square \square}\left\{\sum_{n=1}^{\infty} \frac{K_{w}}{n^{2}} \cdot \sin \left(n p \frac{\alpha}{2}\right) \times \cos \left(n p \theta+n p K \alpha-n p\left(\theta_{0}+q \frac{2 \pi}{3 p}\right)\right)\right. \\
& \left.+\frac{p \delta_{d}}{2} \sum_{h=1}^{\infty} \frac{K_{w}}{n(n p+v)} \cdot \sin \left((n p+v) \frac{\alpha}{2}\right) \times \cos \left(n p \theta+(n p+v) K \alpha-h p\left(\theta_{0}+q \frac{2 \pi}{3 p}\right)\right)\right\}
\end{aligned}
$$

with: $\left\{M_{s r \square \square}=\frac{\mu_{0} r l}{g_{0}} \frac{4 N_{t}}{\pi p^{2}}\right.$ and $v= \pm 1$

Where: $\mathrm{q}=0,1$ and 2 is the degree of DE , $\delta_{\mathrm{d}}$ is the order of harmonic, $\mathrm{K}_{\mathrm{W}}$ is the winding factor, $\mathrm{N}_{\mathrm{t}}$ number of stator turns in series, $g_{0}$ is the air gap length in machine with uniform air gap, 1 is the length of stack, $r$ is the average radius of air gap, $\theta$ is the electrical rotor angular position, $\left(\alpha=\frac{2 \pi}{n_{r}}\right)$ is the mechanical angle of a rotor loops, and $\mathrm{K}$ is a positive integer.

On the other hand, the rotor flux in vector matrix form can be written as:

$$
\left[\psi_{r k}\right]=\left[M_{s r}(\theta)\right]^{t}\left[I_{s a b c}\right]+\left[L_{r}\right]\left[I_{r k}\right]
$$

For an ideal three-phase supply, the stator's currents which flow in the stator's phases are given by:

$$
\left[I_{s a b c}\right]=I_{s m} \cos \left(\omega_{s} t-\mathrm{q} \frac{2 \pi}{3}\right)
$$

Substituting (3) and (5) in (4) leads to the expression of the cage rotor flux:

$$
\begin{array}{r}
{\left[\psi_{r k}\right]=\left[L_{r}\right]\left[I_{r k}\right]+\frac{1}{2} I_{s m} \frac{4 \mu_{0} r l N_{t} k_{w n}}{g_{0} \pi(p h)^{2}} \cdot\left\{\sum_{n=1}^{\infty} M_{s r} \sin \frac{n p \alpha}{2} \cdot\left[\begin{array}{c}
\cos \left(S_{n} \omega_{s} t+n p K \alpha-n p \theta_{0}\right) \\
\vdots \\
\vdots
\end{array}\right]\right.} \\
\left.+\sum_{n=1}^{\infty} \frac{\delta_{d} n p}{2(n p+v)} \cdot \sin \left(\frac{(n p+v) \alpha}{2}\right) \cdot\left[\begin{array}{c}
\cos \left(S_{n} \omega_{s} t+(n p+v) K \alpha-n p \theta_{0}\right) \\
\vdots
\end{array}\right]\right\}
\end{array}
$$

The cage rotor flux described by (6) generates voltages in the rotor. These generated voltages induce the following rotor loops currents:

$$
\left[I_{r k}\right]=\left[I_{r k 1}\right]+\left[I_{r k 2}\right]+\left[I_{r k 3}\right]
$$

where:

$$
\begin{aligned}
& {\left[I_{r k 1}\right]=\sum_{n=1}^{\infty} I_{r k}^{n p}\left[\begin{array}{c}
\cos \left(S_{n} \omega_{s} t+n p K \alpha-n p \theta_{0}\right) \\
\vdots
\end{array}\right]_{K=0,1 \ldots, n_{r}-1}} \\
& {\left[I_{r k 2,3}\right]=\sum_{n=1}^{\infty} I_{r k}^{n p+v}\left[\begin{array}{c}
\cos \left(S_{n} \omega_{s} t+(n p+v) K \alpha-n p \theta_{0}\right) \\
\vdots
\end{array}\right]_{K=0, n_{r}-1}}
\end{aligned}
$$

with : $S_{n}=n(1-s) \pm 1$

\section{GENERATION PROCESS OF HARMONICS IN STATOR CURRENT IN PRESENCE OF DE AND BROKEN ROTOR BARS}

Under DE and Broken rotor bars the stator flux derivative of phase (a) can be written as:

$$
\frac{d\left[\psi_{s a}\right]}{d t}=\left[L_{s}\right] \frac{d\left[I_{s a}\right]}{d t}+\frac{d\left[\phi_{s a 1}\right]}{d t}+\frac{d\left[\phi_{s a 2}\right]}{d t}
$$


where:

$$
\begin{aligned}
& \frac{d\left[\phi_{s a 1}\right]}{d t}=\frac{d\left(\left[M_{s r}(n p)\right] \times\left[I_{r k 1}\left(n^{\prime} p\right)\right]\right)}{d t} \\
& \frac{d\left[\phi_{s a 2,3}\right]}{d t}=\frac{d\left(\left[M_{s r}(n p)\right] \times\left[I_{r k 2,3}\left(n^{\prime} p\right)\right]\right)}{d t}
\end{aligned}
$$

In order to distinguish different terms, we introduced the parameter $\left(n^{\prime}\right)$, which plays the same role as $n$. Now, if we focus our attention, on the term $d \phi_{s a 1} / d t$ we can write $\left[M_{s r}(n p)\right]$ and $\left[I_{r k 1}\left(n^{\prime} p\right)\right]$ by replacing by their expressions

$$
\frac{d \phi_{s a 1}}{d t}=\sum_{n^{\prime}=6 \eta_{ \pm}}^{\omega} \sum_{n=6 \eta_{ \pm 1}}^{\omega} \frac{M_{s r} k_{w}}{2 . n^{2}} \sin \left(n p \frac{\alpha}{2}\right) \times\left\{S_{n_{b}}^{n p}+n \frac{p \delta_{d}}{2} S_{n_{b}}^{n p+v}\right\}
$$

With :

$$
\begin{aligned}
& S_{n_{b}}^{n p}=\sum_{k=0}^{n_{r}-1} I_{r k}^{n p} \times\left\{S_{n^{\prime}} \cdot \omega_{s} \times\left(\sin \left(n p \theta-S_{n^{\prime}} \omega_{s} t-\left(n-n^{\prime}\right) p \theta_{0}+\left(n-n^{\prime}\right) p K \alpha\right)\right.\right. \\
& \left.-\sin \left(n p \theta+S_{n^{\prime}} \omega_{s} t-(n+n) p \theta_{0}+\left(n+n^{\prime}\right) p K \alpha\right)\right) \\
& -\mathrm{np} \frac{\mathrm{d} \theta}{\mathrm{dt}}\left(\sin \left(n p \theta+S_{n^{\prime}} \omega_{s} t-\left(n+n^{\prime}\right) p \theta_{0}+(n+n) p K \alpha\right)\right. \\
& \left.\left.+\sin \left(n p \theta-S_{n^{\prime}} \omega_{s} t-\left(n-n^{\prime}\right) p \theta_{0}+\left(n-n^{\prime}\right) p K \alpha\right)\right)\right\} \\
& S_{n_{b}}^{n p+v}=\sum_{k=0}^{n_{r}-1} I_{r k}^{n p+v} \times\left\{S_{n^{\prime}} \cdot \omega_{s} \times\left(\sin \left(n p \theta-S_{n^{\prime}} \omega_{s} t-\left(n-n^{\prime}\right) p \theta_{0}+\left(n p+v-n^{\prime} p\right) K \alpha\right)\right.\right. \\
& \left.-\sin \left(n p \theta+S_{n^{\prime}} \omega_{s} t-\left(n+n^{\prime}\right) p \theta_{0}+\left(n p+v+n^{\prime} p\right) K \alpha\right)\right) \\
& -\frac{\mathrm{d} \theta}{\mathrm{dt}}\left(\sin \left(n p \theta+S_{n^{\prime}} \omega_{s} t-\left(n+n^{\prime}\right) p \theta_{0}+\left(n p+v+n^{\prime} p\right) K \alpha\right)\right. \\
& \left.+\sin \left(n p \theta-S_{n^{\prime}} \omega_{s} t-\left(n-n^{\prime}\right) p \theta_{0}+\left(n p+v-n^{\prime} p\right) K \alpha\right)\right\}
\end{aligned}
$$

\subsection{DE without broken rotor bars}

In the case of DE without broken rotor bars the amplitude of the rotor loop currents are equal. As a result:

- $\quad \mathrm{S}_{\mathrm{n}_{\mathrm{b}}}^{\mathrm{np}}$ is always null except whenever: $n=\left(\frac{\lambda n r}{p} \pm n^{\prime}\right)$.

- $\quad \mathrm{S}_{\mathrm{n}_{\mathrm{b}}}^{\mathrm{np}+v}$ is always null except whenever: $n=\left(\frac{\lambda n r+v}{p} \pm n^{\prime}\right)$

Under these conditions, induce voltages in stator; these induced voltages will produce a series of high frequency compo-

nents of stator current. Substituting $S_{n}$, by its corresponding expression and $\theta$ by its value $\left(\frac{1-s}{p}\right) \omega_{s} t$, we obtain the expression of these components:

$$
f_{d e}(\lambda)=\left(\frac{\lambda n_{r}}{p}(1-s) \pm 1\right) f_{s}
$$

Following similar reasoning, $\frac{d \phi_{s a 1}}{d t}$ we can prove that induces additional components in the spectrum of stator current All harmonic components generated in the stator current spectrum due to the combined faults can be summarized in Table 1 (for $\left(n^{\prime}=1\right)$ ). It is important to note that these harmonic cannot exists except whenever $n$ belongs to the following set:

$$
C=\left\{\left(n=1 \cup n=\left(\frac{\lambda n_{r}}{p} \pm 1\right) \cup n=\left(\frac{\lambda n_{r} \pm 1}{p} \pm 1\right) \cup n=\left(\frac{n p \pm 2}{p} \pm 1\right)\right)\right\}
$$


Table 1 Stator current frequency components due to DE predicted theoretically and their orders.

\begin{tabular}{|c|c|}
\hline Order $\mathrm{n}$ & Frequency \\
\hline$\frac{\lambda n_{r}}{p}-v$ & $\left(\frac{\lambda n_{r}}{p}(1-s)-v\right) f_{s}$ \\
\hline$\frac{\lambda n_{r}+1}{p}+v$ & $\left(\frac{\lambda n_{r}+1}{p}(1-s)+v\right) f_{s}$ \\
\hline$\frac{\lambda n_{r}-1}{p}+v$ & $\left(\frac{\lambda n_{r}-1}{p}(1-s)+v\right) f_{s}$ \\
\hline$\frac{\lambda n_{r}+2}{p}+v$ & $\left(\frac{\lambda n_{r}+2}{p}(1-s)+v\right) f_{s}$ \\
\hline$\frac{\lambda n_{r}-2}{p}+v$ & $\left(\frac{\lambda n_{r}-2}{p}(1-s)+v\right) f_{s}$ \\
\hline
\end{tabular}

Figure 1 shows the simulated spectral content of the stator current of the machine under healthy condition and with $35 \%$ of DE.
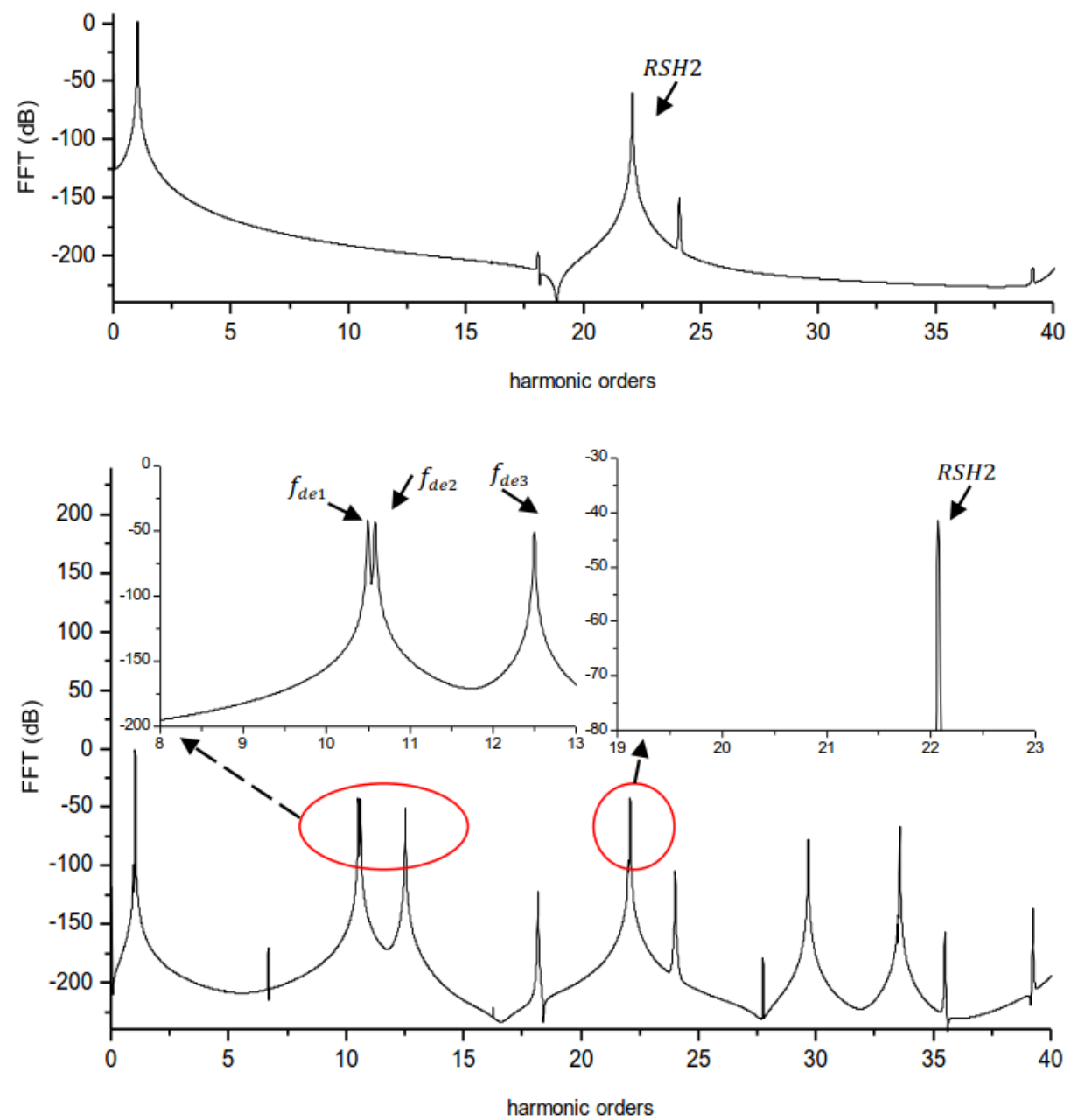

Figure 1. Spectrum of stator current of healthy induction machine.(Top) of machine with DE (Bottom)

The different simulation results in this paper were made for a 1.1-kW motor with 24 stator and 22 rotor slots, two pair poles, and $s=0.0427$. It is easy to verify the total agreement between theoretical formulas (Table1 and (17)) and the simulation results. Unlike the case where the machine is healthy, when DE occurs,

$$
\begin{aligned}
& f_{d e 1}=\left(\frac{22+2}{2}(1-s)+1\right) f_{s}=624.8 \mathrm{~Hz}, f_{d e 2}=\left(\frac{22+2}{2}(1-s)-1\right) f_{s}=524.8 \mathrm{~Hz} \text { and } f_{d e 3}= \\
& \left(\frac{22-2}{2}(1-s)+1\right) f_{s}=529
\end{aligned}
$$


and are present on the spectrum of the stator current.

\subsection{DE with broken rotor bars}

Fault on the rotor, such as cracked rotor bars, causes asymmetrical working condition within the rotor. If the bar between loop $\mathrm{k}$ and $\mathrm{k}+1$ is broken, the current $I_{\mathrm{rk}}$ will be equal to $I_{\mathrm{rk}+1}$, which means that the $I_{\mathrm{rk}}$ is flowing in a double width loop (the maximum values of $I_{r k}$ is larger than the others). So, for each rotor's loop, the rotor current will have amplitude different than the others $\mathrm{I}_{\mathrm{rk}} \neq \mathrm{I}_{\mathrm{rm}}$ bars. In this respect, $S_{n_{b}}^{n p}$ is not equal to zero whatever the value of. Therefore the stator current contains additional harmonic components given by the following equations:

$$
f_{d b}=\left(n(1-s) \pm S_{h^{\prime}}\right) f_{s}
$$

Simulation in figure 2 confirms the precedent expression. We note that the current spectrum reacts to the simultaneous presence of broken rotor bars and DE by showing the well-known characteristic frequencies of broken bars, and harmonics related to DE. More important, it is clear that frequencies of the components caused by the DE are similar to some of the frequencies introduced by the broken bars.

For example:

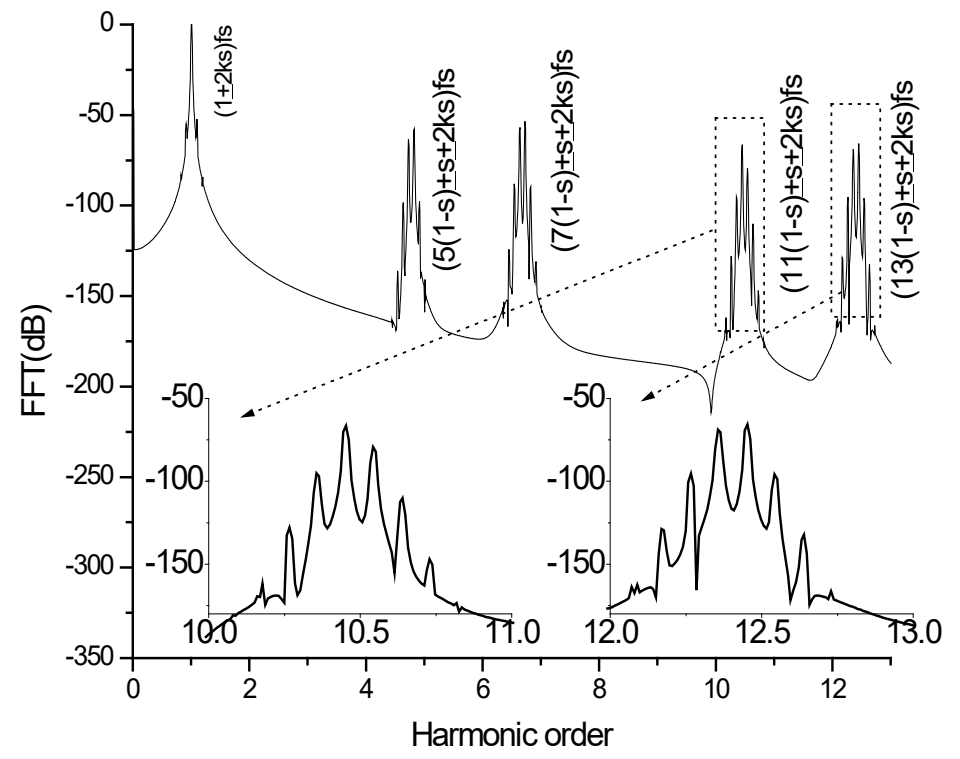

Figure 2. Spectrum of stator line current of an induction machine with one broken bar and 35\% of DE

- The component $f_{d e 1}=\left(\frac{\lambda n_{r}+2}{p}(1-s)+1\right) f_{s}$ related to DE coincides with $(13(1-s)+s) f_{s}=$ $624 \mathrm{~Hz}$ related to rotor broken bars.

- The component $f_{d e 2}=\left(\frac{\lambda n_{r}+2}{p}(1-s)-1\right) f_{s}$ related to DE coincides with $(11(1-s)-s) f_{s}=$ $524 \mathrm{~Hz}$ related to rotor broken bars.

Furthermore, the consideration of speed ripples produce on the sidebands of each existent harmonic and at equal distance $\left( \pm 2 \eta s f_{s}\right)$ new additional harmonic components

\section{LABORATORY TEST SETUP AND EXPERIMENTAL VERIFICATION}

The Tested machine for both healthy and faulty one (one broken bar) is a $1.1 \mathrm{~kW}$ three-phase induction machine FIMET manufacturer with 24 stator and 22 rotor slots, 2 pair poles. In this paper, the original motor's bearing, has been replaced with a very aged bearing where additional free space exists between the balls and the bearing's inner and outer races. This free space introduces the DE (Figure 3). 


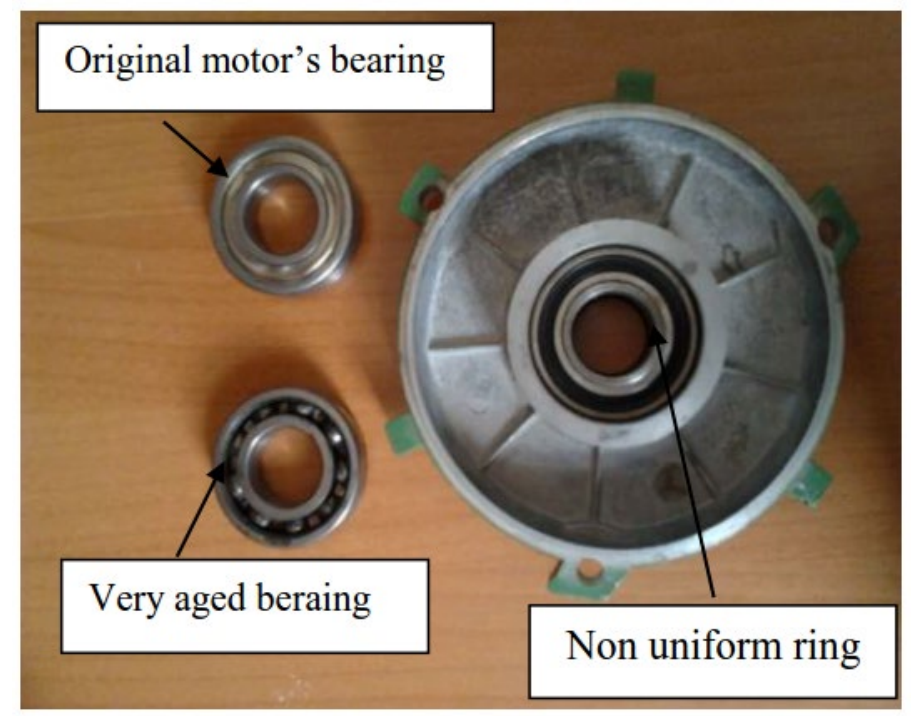

Figure 3. Artificially created DE in induction motor

Figure 4.top presents the spectrum of the stator current for healthy machine operating with a slip s=0.042. Although theoretically $, f_{d e 1}, f_{d e 2}$ and $f_{d e 3}$ would not be present for the motor, one can notice the appearance of $f_{d e 2}$ with a smaller amplitudes. The existence of this component in the experimental spectrum is due to machine constructional asymmetry.

The spectrum of the stator current for machine working with DE is shown in Figure 4.middle. One can notice that $f_{d e 1}$ and $f_{d e 3}$ appear in the stator current and the amplitude of $f_{d e 2}$ shows an increase of approximately $10(-70.2$ to -60.53$)$. The occurrence of $f_{d e 1}$ and $f_{d e 3}$ the difference between the amplitude of $f_{d e 2}$ can be utilized to recognize DE fault. 

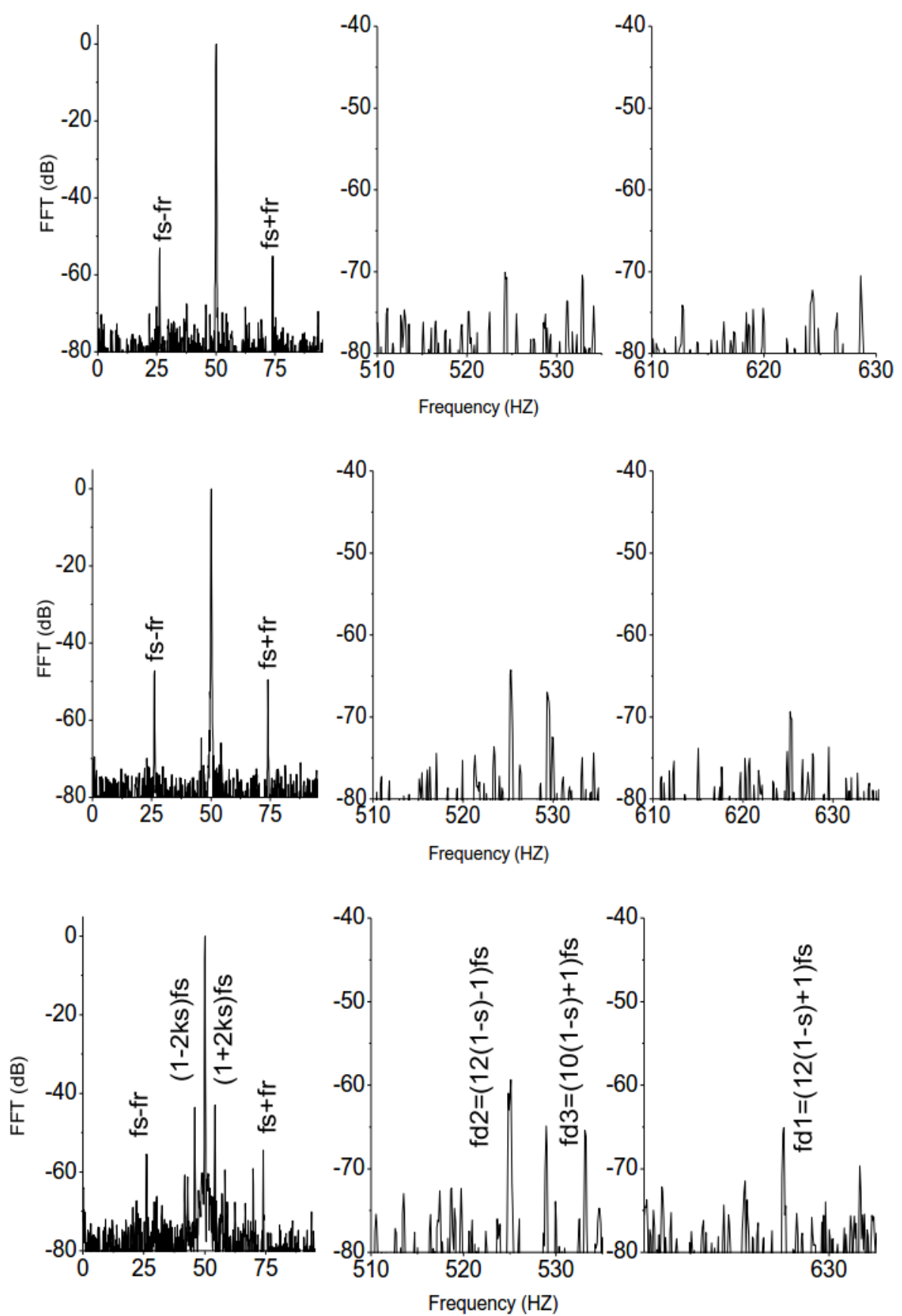

Figure. 4. Experimentally, normalized FFT spectrum of stator line current of an induction machine. Top: Healthy, middle: with DE, bottom: with one broken bar and DE.

Figure 4.bottom shows the spectrum of the stator current for machine working with one broken bar and DE. The main important difference of this spectrum in comparison to the one of the machine with DE is the appearance of the harmonic components related to the broken bar faults given by (1) .

More important the amplitude of $f_{d e 1}, f_{d e 2}$ and $f_{d e 3}$ show an increase of approximately 5 (-70 to -65$), 4$ (-64.2 to -60.53$)$ and $3(-67.1$ to 64.2$) \mathrm{dB}$ respectively.

\section{CONCLUSION}

In this paper, the effects of combined DE and broken rotor bars on the harmonic content of stator current of induction machine have been investigated. A comprehensive mathematical proof of existence of different harmonic components in the stator current spectrum has been presented respectively for machine with DE and machine with mixed faults. The theoretical consideration and the simulation results have been validated on experimental signal. It has been proved that the stator current spectrum reacts to the simultaneous presence of broken bars and DE by showing the well-known 
characteristic frequencies of broken bars, and harmonics related to DE. Since frequencies of the components caused by the $\mathrm{DE}$ are similar to some of the frequencies introduced by the broken bars, we can conclude that on the contrary of broken bars diagnosis, DE diagnosis is very difficult if only stator current analysis is used.

\section{REFERENCES}

[1] Kliman G.B. and Stein J., (1992) "Method of current signature analysis," Electric Machines and Power Systems, vol. 20, no. 5 pp. $463-474$.

[2] Kliman G.B., Koegl R.A. and J. Stein and M.W. Madden,(1988) "Noninvasive detection of broken rotor bars in operating induction motors", IEEE Transactions on Energy Conversion, vol. EC-3, no. 4,pp.873-879.

[3] Benbouzid M. E. H. (2000) "A review of induction motors signature analysis as a medium for faults detection," IEEE Transactions on Industrial Electronics, vol. 47, pp. 984-993

[4] FilliPPetti F., Franceschini G., Tassoni C. and Vas P., (1996) "AI thechnique in induction machine diagnosis including the speed ripple effect”, IEEE-IAS, California, , vol. 1, pp. 655 - 662.

[5] Faiz J., Ebrahimi B.M., Akin B. and Toliyat H.A., (2009) "Comprehensive Eccentricity Fault Diagnosis in Induction Motors Using Finite Element Method "IEEE Transactions on Magnetics, vol. 45 , no. 3, pp. 1764-1767.

[6] Cameron R., Thomson W.T. and Dow A.B Vibration and current monitoring for detecting airgap eccentricity in large induction motors", IEE Proceedings-B May 1986, vol. 133, no. 3 , pp. 155-163.

[7] Nandi S., Ahmed S., and Toliyat H.A.,(2001) "Detection of Rotor Slot and Other Eccentricity Related Harmonics in a Three Phase Induction Motor with Different Rotor Cages," IEEE Transactions on Energy Conversion, vol. 16, no. 3, pp. 253-260.

[8] Joksimovic G., (2005) "Dynamic simulation of cage induction machine with air gap eccentricity, IEE Proc. Electr. Power Appl. 152 (4), pp. 803-810.

[9] Kaikaa M. , Hadjami M. , and khezzar A. , (2014) "Effects of the Simultaneous Presence of Static Eccentricity and Broken Rotor Bars on the Stator Current of Induction Machine," IEEE Trans. Industrial Electronics, vol. 61, $\mathrm{n}^{\circ}: 5$, pp $2452-2463$.

[10] Kaikaa M.Y. (2014) "Effect of unbalanced supply on dynamic eccentricity fault diagnosis in induction motor," International Journal of Applied Electromagnetics and Mechanics Volume 46, Number 4,Pages 793-808

[11] Dorrell D.G.,. Thomson W.T and Roach S., (1997) "Analyse of air-gap flux, current, and vibration signals as function of combination static and dynamic air-gap eccentricity in 3-phase induction motors," IEEE Trans. Industry Applications, vol. 33, no. 1, pp. 24-34.

[12] Khezzar A., Hadjami M., Bessous N., Oumaamar, M.E.K. and Razik H., (2008) "Accurate Modelling of Cage Induction Machine with Analytical Evaluation of Inductances" 34th Annual Conference of the IEEE Industrial Electronics Society, IECON'08, Orlando, Floride, USA, 10-13 Nov. 2008, pp. 1112 - 1117. 